\section{Effect of Nitrogen Fertilization Rate on Aesthetic Quality of Landscape-grown Vines and Groundcovers}

\author{
Amy L. Shober ${ }^{1,7}$, Kimberly A. Moore ${ }^{2}$, Gitta S. Hasing ${ }^{3}$, \\ Christine Wiese ${ }^{4}$, Geoffrey C. Denny ${ }^{5}$, and Gary W. Knox ${ }^{6}$
}

ADDITIONAL INDEX WORDS. landscape maintenance, ornamental plants

SUMMARY. Research supporting recommendations for fertilizer needs of landscapegrown vines and groundcovers is lacking. The objectives of our study were to (1) evaluate the quality response of selected vine and groundcover species to nitrogen (N) fertilization at five rates and (2) validate the recommended $\mathrm{N}$ fertilizer rates (from the initial evaluation) by monitoring quality of additional landscape-grown vine and groundcover species. Three vine species and two groundcover species were planted in west-central Florida into raised beds containing subsoil fill material in a completely randomized design. Plants were fertilized every 6 weeks with a controlled release fertilizer $(20 \mathrm{~N}-0 \mathrm{P}-0 \mathrm{~K}-23 \mathrm{~S})$ at an annual $\mathrm{N}$ rate of $0,2,4,6$, or $12 \mathrm{lb} / 1000 \mathrm{ft}^{2}$. Plant aesthetic quality (0-5 scale) was assessed every 6 weeks for 30 weeks after planting. Although quality of some species increased significantly as $\mathbf{N}$ rate increased, all plants supplied with at least $4 \mathrm{lb} / 1000 \mathrm{ft}^{2}$ per year $\mathrm{N}$ fertilizer had acceptable quality ratings of 3 or better. Screening of three additional vines and four additional groundcovers fertilized with controlled release fertilizer $(42 \mathrm{~N}-0 \mathrm{P}-0 \mathrm{~K})$ at an annual $\mathrm{N}$ rate of 3,5 , or $7 \mathrm{lb} / 1000 \mathrm{ft}^{2}$ confirmed that fertilization with 2 to 4 $\mathrm{lb} / 1000 \mathrm{ft}^{2}$ per year should be adequate to maintain acceptable vines and groundcovers grown in the landscape in west-central Florida.

$\mathrm{T}$ he University of Florida-Institute of Food and Agricultural Sciences (UF-IFAS) Florida Friendly Landscaping ${ }^{\mathrm{TM}}$ Program (FFL) provides general $\mathrm{N}$ fertilizer recommendations for ornamental landscape plants that range from 0 to $6 \mathrm{lb} / 1000$ $\mathrm{ft}^{2}$ of $\mathrm{N}$ annually depending on desired maintenance level (FFL, 2009). However, the current FFL-recommended rates are applied broadly overall plant

The work was supported by the Florida Agricultural Experiment Station, the Southwest Florida Water Management District, and the Florida Nursery Growers and Landscape Association.

${ }^{1}$ Associate Professor, Department of Plant and Soil Sciences, University of Delaware, 531 South College Avenue, Newark, DE 19716

${ }^{2}$ Professor, Environmental Horticulture Department, Fort Lauderdale Research and Education Center, Institute of Food and Agriculture Science, University of Florida, 3205 College Avenue, Davie, FL 33314

${ }^{3}$ Biologist, Environmental Horticulture Department, Gulf Coast Research and Education Center, Institute of Food and Agriculture Science, University of Florida, 14625 CR 672, Wimauma, FL 33598

${ }^{4}$ Biologist, Environmental Horticulture Department, Institute of Food and Agriculture Science, University of Florida, 106 Building 68, Gainesville, FL 32611

${ }^{5}$ Assistant Extension Professor, Mississippi State University, 246 Dorman Hall, Mississippi State, MS 39762

${ }^{6}$ Professor, Environmental Horticulture Department, North Florida Research and Education Center, Institute of Food and Agriculture Science, University of Florida, 155 Research Road, Quincy, FL 32351

${ }^{7}$ Corresponding author. E-mail: ashober@udel.edu. types or species. Previous research suggested that $\mathrm{N}$ fertilizer needs of ornamental plants in Florida landscapes vary by plant type and species (Shober et al., 2013; Shurberg et al., $2012 a, b)$. The authors suggested that application of $\mathrm{N}$ fertilizer at a rate of 4 to $6 \mathrm{lb} / 1000 \mathrm{ft}^{2}$ per year was adequate to produce acceptable quality warm and cool season annuals in the landscape (Shurberg et al., 2012b), whereas acceptable quality herbaceous perennials (Shurberg et al., 2012a) and woody shrubs (Shober et al., 2013) could be maintained with annual $\mathrm{N}$ applications of 2 to $4 \mathrm{lb} / 1000 \mathrm{ft}^{2}$.

It is uncertain whether landscapegrown vines and groundcovers will require $\mathrm{N}$ fertilizer inputs as common annuals, perennials, or shrubs. Most research evaluating vine or groundcover response to $\mathrm{N}$ fertilizer rate was conducted in a nursery production (Berberich et al., 2006; Dumroese, 2003; Holcomb et al., 1992) or fruit crop production setting (Santos and Gilreath, 2006; Utsunomiya et al., 1998). Research supporting recommendations for the fertilizer needs of landscape-grown vines and groundcovers is lacking. The few $\mathrm{N}$ fertilizer recommendations for vines and groundcovers reported in the literature are often very general and variable. For example, Park-Brown and Knox (2010) noted that most vine species can benefit from $\mathrm{N}$ fertilization during establishment, but many species require little to no $\mathrm{N}$ fertilizer once established. Henley and Black (2003) recommended application of $\mathrm{N}$ at an annual rate of 2 to $3 \mathrm{lb} / 1200 \mathrm{ft}^{2}$ for english ivy (Hedera helix) grown outdoor in Florida to maintain "moderate vigor." The objectives of our study were to (1) evaluate the quality response of selected vine and groundcover species to $\mathrm{N}$ fertilization at five rates and (2) validate the recommended $\mathrm{N}$ fertilizer rates (from the initial evaluation) by monitoring quality of additional landscape-grown vine and groundcover species.

\section{Materials and methods}

Evaluation OF $\mathbf{N}$ FerTilizer REQUIREMENTS. Three vine species [coral honeysuckle (Lonicera sempervirens), cross vine (Bignonia capreolata), and pipe vine (Aristolochia sp.)] and two groundcovers [society garlic (Tulbaghia violacea) and african iris (Dietes bicolor)] were selected for evaluation of quality response to five annual $\mathrm{N}$ fertilizer rates $(0,2,4,6$, and $12 \mathrm{lb} / 1000 \mathrm{ft}^{2}$ ) when grown in the landscape. All plant materials were received in 1-gal containers from Amplex (Clearwater, FL).

Raised beds $(5 \times 200 \times 0.5 \mathrm{ft})$ were constructed with subsoil fill material (St. John's fine sand, Sandy, siliceous,

\begin{tabular}{lllc}
\hline $\begin{array}{l}\text { Units } \\
\text { To convert U.S. to SI, } \\
\text { multiply by }\end{array}$ & U.S. unit & SI unit & $\begin{array}{l}\text { To convert SI to U.S., } \\
\text { multiply by }\end{array}$ \\
\hline 0.3048 & $\mathrm{ft}$ & $\mathrm{m}$ & 3.2808 \\
0.0929 & $\mathrm{ft}^{2}$ & $\mathrm{~m}^{2}$ & 10.7639 \\
3.7854 & $\mathrm{gal}$ & $\mathrm{L}$ & 0.2642 \\
0.4075 & $\mathrm{gal} / 100 \mathrm{ft}^{2}$ & $\mathrm{~L} \cdot \mathrm{m}^{-2}$ & 2.4542 \\
2.54 & inch $(\mathrm{es})$ & $\mathrm{cm}$ & 0.3937 \\
0.4536 & $\mathrm{lb}$ & $\mathrm{kg}$ & 2.2046 \\
48.8243 & $\mathrm{lb} / 1000 \mathrm{ft}^{2}$ & $\mathrm{~kg} \cdot \mathrm{ha}^{-1}$ & 0.0205
\end{tabular}


hyperthermic Typic Alaquods) [U.S. Department of Agriculture (USDA), 2004] at the UF-IFAS Gulf Coast Research and Education Center in Wimauma, FL (USDA hardiness zone 9b). An initial sample of soil fill was analyzed for $\mathrm{pH}$ and soil nutrient content before transplanting; methods and results were reported previously (Shurberg et al., 2012a,b). Raised beds were divided into 19 plots $(20 \times 5 \mathrm{ft})$. Two plants of each vine species and three plants of each groundcover species were planted into each plot on 10 Dec. 2008. Vines were spaced $3 \mathrm{ft}$ apart and supported by a trellis (five horizontal wires spaced $\mathrm{l} \mathrm{ft}$ apart to a height of $5 \mathrm{ft}$ ); groundcovers were spaced evenly in two alternating rows along each plot.

Irrigation was applied to raised beds and field plots through four drip lines (Jain Irrigation, Winter Haven, $\mathrm{FL}$ ) that were placed on $\mathrm{l}$-ft spacing; emitters were spaced 8 inches apart. The flow rate of the drip line was 0.65 $\mathrm{gal} / 100 \mathrm{ft}^{2}$ per minute. Vines and groundcovers were irrigated three times per week at $0830 \mathrm{HR}$ for $25 \mathrm{~min}$ per irrigation event. Weeds were removed by hand or sprayed with glyphosate (Round-Up ${ }^{\circledR}$; Monsanto, Creve Coeur, MO) as needed. No mulch was added to beds to minimize outside $\mathrm{N}$ contributions. Vines and groundcovers were fertilized every 6 weeks (based on the release rate of fertilizer published by the manufacturer) with a $20 \mathrm{~N}-0 \mathrm{P}-0 \mathrm{~K}-23 \mathrm{~S}$ polymer-coated ammonium sulfate fertilizer (Honeywell nylon, Seffner, FL) at $0,2,4,6$, or $12 \mathrm{lb} / 1000 \mathrm{ft}^{2}$ per year.

Vines and groundcovers were rated visually for aesthetic quality every 6 weeks for a period of 54 weeks after planting (WAP). Visual quality ratings were assigned on a 0 to 5 scale by considering canopy dieback, canopy density, flower coverage (if applicable), and form as follows: $0=$ dead, $\mathrm{l}=$ poor (low canopy density, few to no flowers, widespread chlorosis), 2 = below average (significant dieback, chlorosis, low canopy density, poor flowering), 3 = average (moderate dieback, good form, adequate flowering, little to no chlorosis), $4=$ above average (minimal dieback, no noticeable chlorosis, above average density, many flowers), and $5=$ excellent (dense leaf canopy, many flowers, no visible nutrient deficiencies, no
Table 1. Average plant quality response of selected landscape grown vine species to controlled release nitrogen $(\mathrm{N})$ applied at five rates planted $10 \mathrm{Dec} .2008$ in west-central Florida (U.S. Department of Agriculture hardiness zone 9b).

\begin{tabular}{|c|c|c|c|}
\hline \multirow{2}{*}{$\begin{array}{l}\text { WAP and fertilizer } \\
\mathrm{N} \text { rate }\left(1 \mathrm{~b} / 1000 \mathrm{ft}^{2}\right)^{\mathrm{z}}\end{array}$} & \multicolumn{3}{|c|}{ Plant aesthetic quality $(0-5 \text { scale })^{\mathrm{y}}$} \\
\hline & Pipe vine & Cross vine & Coral honeysuckle \\
\hline \multicolumn{4}{|l|}{$6 \mathrm{WAP}$} \\
\hline 0 & $3.3 \mathrm{a}^{\mathrm{x}}$ & $2.7 \mathrm{a}$ & $3.5 \mathrm{a}$ \\
\hline 2 & $3.3 \mathrm{a}$ & $2.3 \mathrm{a}$ & $3.5 \mathrm{a}$ \\
\hline 4 & $2.6 \mathrm{a}$ & $2.5 \mathrm{a}$ & $3.6 \mathrm{a}$ \\
\hline 6 & $3.1 \mathrm{a}$ & $2.9 \mathrm{a}$ & $3.6 \mathrm{a}$ \\
\hline 12 & $3.0 \mathrm{a}$ & $2.8 \mathrm{a}$ & $4.0 \mathrm{a}$ \\
\hline \multicolumn{4}{|l|}{12 WAP } \\
\hline 0 & $1.3 \mathrm{a}$ & $3.3 \mathrm{a}$ & $2.7 \mathrm{a}$ \\
\hline 2 & $1.1 \mathrm{a}$ & $3.1 \mathrm{a}$ & $2.9 \mathrm{a}$ \\
\hline 4 & $1.1 \mathrm{a}$ & $3.2 \mathrm{a}$ & $3.1 \mathrm{a}$ \\
\hline 6 & $1.1 \mathrm{a}$ & $3.3 \mathrm{a}$ & $2.5 \mathrm{a}$ \\
\hline 12 & $0.8 \mathrm{a}$ & $3.4 \mathrm{a}$ & $4.5 \mathrm{a}$ \\
\hline \multicolumn{4}{|l|}{$18 \mathrm{WAP}$} \\
\hline 0 & $2.0 \mathrm{a}$ & $3.0 \mathrm{a}$ & $2.0 \mathrm{a}$ \\
\hline 2 & $2.5 \mathrm{a}$ & $3.3 \mathrm{a}$ & $2.6 \mathrm{a}$ \\
\hline 4 & $1.9 \mathrm{a}$ & $3.4 \mathrm{a}$ & $2.9 \mathrm{a}$ \\
\hline 6 & $1.9 \mathrm{a}$ & $3.4 \mathrm{a}$ & $2.6 \mathrm{a}$ \\
\hline 12 & $1.0 \mathrm{a}$ & $3.5 \mathrm{a}$ & $3.9 \mathrm{a}$ \\
\hline \multicolumn{4}{|l|}{$24 \mathrm{WAP}$} \\
\hline 0 & $2.2 \mathrm{a}$ & $3.2 \mathrm{~b}$ & $2.0 \mathrm{a}$ \\
\hline 2 & $2.4 \mathrm{a}$ & $3.5 \mathrm{a}$ & $2.5 \mathrm{a}$ \\
\hline 4 & $2.3 \mathrm{a}$ & $3.3 \mathrm{ab}$ & $2.3 \mathrm{a}$ \\
\hline 6 & $2.1 \mathrm{a}$ & $4.4 \mathrm{ab}$ & $2.3 \mathrm{a}$ \\
\hline 12 & $1.3 \mathrm{a}$ & $4.6 \mathrm{a}$ & $3.9 \mathrm{a}$ \\
\hline \multicolumn{4}{|l|}{$30 \mathrm{WAP}$} \\
\hline 0 & $1.8 \mathrm{a}$ & $2.3 \mathrm{~b}$ & $1.7 \mathrm{a}$ \\
\hline 2 & $2.0 \mathrm{a}$ & $3.0 \mathrm{ab}$ & $1.4 \mathrm{a}$ \\
\hline 4 & $1.9 \mathrm{a}$ & $3.1 \mathrm{ab}$ & $0.9 \mathrm{a}$ \\
\hline 6 & $2.4 \mathrm{a}$ & $3.9 \mathrm{a}$ & $1.8 \mathrm{a}$ \\
\hline 12 & $1.4 \mathrm{a}$ & $4.4 \mathrm{a}$ & $0.9 \mathrm{a}$ \\
\hline \multicolumn{4}{|l|}{$36 \mathrm{WAP}$} \\
\hline 0 & $1.5 \mathrm{a}$ & $2.3 \mathrm{c}$ & $0.0 \mathrm{a}$ \\
\hline 2 & $1.9 \mathrm{a}$ & $3.0 \mathrm{c}$ & $0.0 \mathrm{a}$ \\
\hline 4 & $1.9 \mathrm{a}$ & $3.3 \mathrm{bc}$ & $0.0 \mathrm{a}$ \\
\hline 6 & $2.1 \mathrm{a}$ & $4.1 \mathrm{ab}$ & $0.0 \mathrm{a}$ \\
\hline 12 & $1.3 \mathrm{a}$ & $4.5 \mathrm{a}$ & $0.0 \mathrm{a}$ \\
\hline \multicolumn{4}{|l|}{$42 \mathrm{WAP}$} \\
\hline 0 & $1.3 \mathrm{a}$ & $2.7 \mathrm{c}$ & $0.0 \mathrm{a}$ \\
\hline 2 & $1.9 \mathrm{a}$ & $3.1 \mathrm{bc}$ & $0.0 \mathrm{a}$ \\
\hline 4 & $1.4 \mathrm{a}$ & $3.3 \mathrm{bc}$ & $0.0 \mathrm{a}$ \\
\hline 6 & $1.8 \mathrm{a}$ & $4.1 \mathrm{ab}$ & $0.0 \mathrm{a}$ \\
\hline 12 & $1.0 \mathrm{a}$ & $4.5 \mathrm{a}$ & $0.0 \mathrm{a}$ \\
\hline \multicolumn{4}{|l|}{$48 \mathrm{WAP}$} \\
\hline 0 & $1.0 \mathrm{a}$ & $2.3 \mathrm{~b}$ & $0.0 \mathrm{a}$ \\
\hline 2 & $2.0 \mathrm{a}$ & $3.0 \mathrm{ab}$ & $0.0 \mathrm{a}$ \\
\hline 4 & $1.5 \mathrm{a}$ & $3.1 \mathrm{ab}$ & $0.0 \mathrm{a}$ \\
\hline 6 & $1.8 \mathrm{a}$ & $3.5 \mathrm{a}$ & $0.0 \mathrm{a}$ \\
\hline 12 & $0.9 \mathrm{a}$ & $4.0 \mathrm{a}$ & $0.0 \mathrm{a}$ \\
\hline \multicolumn{4}{|l|}{$54 \mathrm{WAP}$} \\
\hline 0 & $1.2 \mathrm{a}$ & $1.8 \mathrm{~d}$ & $0.0 \mathrm{a}$ \\
\hline 2 & $1.8 \mathrm{a}$ & $2.6 \mathrm{~cd}$ & $0.0 \mathrm{a}$ \\
\hline 4 & $1.5 \mathrm{a}$ & $3.0 \mathrm{bc}$ & $0.0 \mathrm{a}$ \\
\hline 6 & $1.5 \mathrm{a}$ & $3.5 \mathrm{ab}$ & $0.0 \mathrm{a}$ \\
\hline 12 & $0.9 \mathrm{a}$ & $3.9 \mathrm{a}$ & $0.0 \mathrm{a}$ \\
\hline
\end{tabular}

${ }^{2} \mathrm{WAP}=$ week after planting; $\mathrm{l} \mathrm{lb} / 1000 \mathrm{ft}^{2}=48.8243 \mathrm{~kg} \cdot \mathrm{ha}^{-1}$.

${ }^{\mathrm{y}} 0=$ dead plant $; 5$ = outstanding plant quality (dense leaf canopy, high-quality flowers, and no nutrient deficiencies or dieback).

${ }^{x}$ Mean separation for each species and WAP by Tukey's honestly significant difference test at $P<0.05$. 
Table 2. Average plant quality response of two landscape-grown groundcover species to controlled release nitrogen $(\mathrm{N})$ applied at five rates planted $10 \mathrm{Dec} .2008$ in west-central Florida (U.S. Department of Agriculture hardiness zone $9 b$ ).

\begin{tabular}{|c|c|c|}
\hline \multirow{2}{*}{$\begin{array}{l}\text { WAP and fertilizer } \\
\mathrm{N} \text { rate }\left(\mathrm{lb} / 1000 \mathrm{ft}^{2}\right)^{\mathrm{z}}\end{array}$} & \multicolumn{2}{|c|}{ Plant aesthetic quality $(0-5 \text { scale })^{y}$} \\
\hline & Society garlic & African iris \\
\hline \multicolumn{3}{|l|}{$6 \mathrm{WAP}$} \\
\hline 0 & $3.7 \mathrm{a}^{\mathrm{x}}$ & $4.4 \mathrm{a}$ \\
\hline 2 & $3.3 \mathrm{a}$ & $5.0 \mathrm{a}$ \\
\hline 4 & $3.4 \mathrm{a}$ & $4.5 \mathrm{a}$ \\
\hline 6 & $3.6 \mathrm{a}$ & $4.4 \mathrm{a}$ \\
\hline 12 & $3.6 \mathrm{a}$ & $4.6 \mathrm{a}$ \\
\hline \multicolumn{3}{|l|}{12 WAP } \\
\hline 0 & $3.0 \mathrm{a}$ & $3.9 \mathrm{a}$ \\
\hline 2 & $3.1 \mathrm{a}$ & $4.3 \mathrm{a}$ \\
\hline 4 & $3.8 \mathrm{a}$ & $3.8 \mathrm{a}$ \\
\hline 6 & $3.4 \mathrm{a}$ & $4.1 \mathrm{a}$ \\
\hline 12 & $3.6 \mathrm{a}$ & $4.1 \mathrm{a}$ \\
\hline \multicolumn{3}{|l|}{$18 \mathrm{WAP}$} \\
\hline 0 & $2.9 \mathrm{~b}$ & $4.4 \mathrm{a}$ \\
\hline 2 & $2.7 \mathrm{c}$ & $4.6 \mathrm{a}$ \\
\hline 4 & $3.4 \mathrm{abc}$ & $3.9 \mathrm{a}$ \\
\hline 6 & $3.7 \mathrm{ab}$ & $4.1 \mathrm{a}$ \\
\hline 12 & $3.8 \mathrm{a}$ & $4.2 \mathrm{a}$ \\
\hline \multicolumn{3}{|l|}{24 WAP } \\
\hline 0 & $3.2 \mathrm{~b}$ & $4.8 \mathrm{a}$ \\
\hline 2 & $3.4 \mathrm{~b}$ & $4.8 \mathrm{a}$ \\
\hline 4 & $3.8 \mathrm{ab}$ & $4.5 \mathrm{a}$ \\
\hline 6 & $3.8 \mathrm{ab}$ & $4.5 \mathrm{a}$ \\
\hline 12 & $4.2 \mathrm{a}$ & $4.3 \mathrm{a}$ \\
\hline \multicolumn{3}{|l|}{$30 \mathrm{WAP}$} \\
\hline 0 & $2.6 \mathrm{a}$ & $4.1 \mathrm{a}$ \\
\hline 2 & $2.6 \mathrm{a}$ & $4.1 \mathrm{a}$ \\
\hline 4 & $2.6 \mathrm{a}$ & $3.6 \mathrm{a}$ \\
\hline 6 & $3.2 \mathrm{a}$ & $3.7 \mathrm{a}$ \\
\hline 12 & $3.0 \mathrm{a}$ & $3.9 \mathrm{a}$ \\
\hline \multicolumn{3}{|l|}{$36 \mathrm{WAP}$} \\
\hline 0 & $2.6 \mathrm{a}$ & $3.9 \mathrm{a}$ \\
\hline 2 & $2.6 \mathrm{a}$ & $4.2 \mathrm{a}$ \\
\hline 4 & $2.8 \mathrm{a}$ & $4.3 \mathrm{a}$ \\
\hline 6 & $2.6 \mathrm{a}$ & $4.4 \mathrm{a}$ \\
\hline 12 & $2.8 \mathrm{a}$ & $4.6 \mathrm{a}$ \\
\hline \multicolumn{3}{|l|}{$42 \mathrm{WAP}$} \\
\hline 0 & $2.3 \mathrm{a}$ & $4.1 \mathrm{a}$ \\
\hline 2 & $2.2 \mathrm{a}$ & $3.9 \mathrm{a}$ \\
\hline 4 & $2.3 \mathrm{a}$ & $4.2 \mathrm{a}$ \\
\hline 6 & $2.4 \mathrm{a}$ & $4.5 \mathrm{a}$ \\
\hline 12 & $2.3 \mathrm{a}$ & $4.5 \mathrm{a}$ \\
\hline \multicolumn{3}{|l|}{48 WAP } \\
\hline 0 & $2.2 \mathrm{a}$ & $3.2 \mathrm{~b}$ \\
\hline 2 & $2.2 \mathrm{a}$ & $3.2 \mathrm{~b}$ \\
\hline 4 & $2.5 \mathrm{a}$ & $3.7 \mathrm{ab}$ \\
\hline 6 & $2.4 \mathrm{a}$ & $3.6 \mathrm{ab}$ \\
\hline 12 & $2.3 \mathrm{a}$ & $3.9 \mathrm{a}$ \\
\hline \multicolumn{3}{|l|}{54 WAP } \\
\hline 0 & $2.3 \mathrm{a}$ & $3.0 \mathrm{c}$ \\
\hline 2 & $2.7 \mathrm{a}$ & $3.2 \mathrm{bc}$ \\
\hline 4 & $2.8 \mathrm{a}$ & $3.7 \mathrm{abc}$ \\
\hline 6 & $2.8 \mathrm{a}$ & $3.7 \mathrm{ab}$ \\
\hline 12 & $2.9 \mathrm{a}$ & $3.9 \mathrm{a}$ \\
\hline
\end{tabular}

${ }^{2} \mathrm{WAP}=$ week after planting; $\mathrm{l} \mathrm{lb} / 1000 \mathrm{ft}^{2}=48.8243 \mathrm{~kg} \cdot \mathrm{ha}^{-1}$.

${ }^{\mathrm{v}} 0=$ dead plant; $5=$ outstanding plant quality (dense leaf canopy, high-quality flowers, and no nutrient deficiencies or dieback).

${ }^{x}$ Mean separation for each species and WAP by Tukey's honestly significant difference test at $P<0.05$. dieback, uniform plant) (Shober et al., 2009).

VALIDATION OF $\mathbf{N}$ FerTilizer RECOMMENDATIONS. Three additional vine species [passion vine (Passiflora incarnate), bower vine (Pandorea jasminoides), and carolina yellow jessamine (Gelsemium sempervirens)] and four additional groundcover species ['Blue Pacific' juniper (Juniperus conferta), sunshine mimosa (Mimosa strigillosa), purple lovegrass (Eragrostis spectabilis), and ornamental sweetpotato (Ipomoea batatas)] were selected to screen the quality of additional plant materials when fertilized at $\mathrm{N}$ rates suggested from the initial evaluation. Cross vine (Bignonia capreolata) were planted in the initial evaluation study and were included in the validation as a reference species to ensure a consistent quality response to the $\mathrm{N}$ fertilizer rates across both studies. Vine species were received in 3 -gal containers and groundcovers were received in 1 -gal containers from Amplex for use in the validation study and were planted into the raised beds on 24 Nov. 2011 following the same experimental design described for the evaluation phase with the following exceptions: bed size was $5 \times 40 \mathrm{ft}$; $42 \mathrm{~N}-0 \mathrm{P}-0 \mathrm{~K}$ slow-release, polymercoated urea fertilizer (Polyon; Harrell's, Lakeland, FL) was applied every 12 weeks (based on the release rate of fertilizer published by the manufacturer) at an annual $\mathrm{N}$ rate of 3,5 , or 7 $\mathrm{lb} / 1000 \mathrm{ft}^{2}$, and quality ratings were assigned over a period of 54 WAP starting at 6 WAP for groundcovers and 12 WAP for vines.

Statistical analysis. The experiments were designed as a completely randomized block design with each fertilizer rate assigned randomly (three replicates) to raised beds containing two (vines) and three (groundcovers) individual plants of each species. Plant aesthetic quality data were analyzed by species and WAP using PROC GLIMMIX (SAS version 9.2; SAS Institute, Cary, NC) with $\mathrm{N}$ rate as a fixed effect. Individual plant $\times$ treatment was included as a random effect using a normal distribution and an identity link function. Pairwise comparisons for aesthetic quality were conducted with the Tukey's honestly significant difference (HSD) test at a significance level of $\alpha=0.05$. Data were checked for normality by examining histogram 
Table 3. Average plant quality response of additional landscape-grown vine species to controlled release nitrogen $(\mathrm{N})$ applied at three rates planted 15 Aug. 2011 in west-central Florida (U.S. Department of Agriculture hardiness zone $9 \mathrm{~b})$ to validate $\mathrm{N}$ fertilizer recommendations.

\begin{tabular}{|c|c|c|c|c|}
\hline \multirow[b]{2}{*}{$\begin{array}{l}\text { WAP and fertilizer } \\
\mathrm{N} \text { rate }\left(\mathrm{lb} / 1000 \mathrm{ft}^{2}\right)^{\mathrm{z}}\end{array}$} & \multicolumn{4}{|c|}{ Plant aesthetic quality $(0-5 \text { scale })^{\mathrm{y}}$} \\
\hline & $\begin{array}{l}\text { Bower } \\
\text { vine }\end{array}$ & $\begin{array}{l}\text { Cross } \\
\text { vine }\end{array}$ & $\begin{array}{c}\text { Carolina } \\
\text { yellow jessamine }\end{array}$ & $\begin{array}{c}\text { Passion } \\
\text { vine }\end{array}$ \\
\hline \multicolumn{5}{|l|}{$12 \mathrm{WAP}$} \\
\hline 3 & $4.7 \mathrm{a}^{\mathrm{x}}$ & $4.0 \mathrm{a}$ & $4.3 \mathrm{a}$ & $4.3 \mathrm{a}$ \\
\hline 5 & $5.0 \mathrm{a}$ & $4.5 \mathrm{a}$ & $4.3 \mathrm{a}$ & $4.2 \mathrm{a}$ \\
\hline 7 & $4.8 \mathrm{a}$ & $4.2 \mathrm{a}$ & $4.0 \mathrm{a}$ & $4.3 \mathrm{a}$ \\
\hline \multicolumn{5}{|l|}{18 WAP } \\
\hline 3 & $3.7 \mathrm{a}$ & $3.5 \mathrm{a}$ & $3.3 \mathrm{a}$ & $3.7 \mathrm{a}$ \\
\hline 5 & $3.8 \mathrm{a}$ & $3.3 \mathrm{a}$ & $3.7 \mathrm{a}$ & $3.5 \mathrm{a}$ \\
\hline 7 & $3.8 \mathrm{a}$ & $3.2 \mathrm{a}$ & $3.0 \mathrm{a}$ & $4.3 \mathrm{a}$ \\
\hline \multicolumn{5}{|l|}{24 WAP } \\
\hline 3 & $3.8 \mathrm{a}$ & $3.0 \mathrm{a}$ & $3.8 \mathrm{a}$ & $4.0 \mathrm{a}$ \\
\hline 5 & $3.8 \mathrm{a}$ & $3.0 \mathrm{a}$ & $3.5 \mathrm{a}$ & $4.0 \mathrm{a}$ \\
\hline 7 & $4.0 \mathrm{a}$ & $3.2 \mathrm{a}$ & $3.6 \mathrm{a}$ & $4.7 \mathrm{a}$ \\
\hline \multicolumn{5}{|l|}{$30 \mathrm{WAP}$} \\
\hline 3 & $3.3 \mathrm{a}$ & $3.7 \mathrm{a}$ & $3.8 \mathrm{a}$ & $3.3 \mathrm{~b}$ \\
\hline 5 & $3.8 \mathrm{a}$ & $4.0 \mathrm{a}$ & $3.8 \mathrm{a}$ & $3.5 \mathrm{~b}$ \\
\hline 7 & $4.0 \mathrm{a}$ & $3.8 \mathrm{a}$ & $4.4 \mathrm{a}$ & $4.5 \mathrm{a}$ \\
\hline \multicolumn{5}{|l|}{$36 \mathrm{WAP}$} \\
\hline 3 & $3.0 \mathrm{a}$ & $3.5 \mathrm{a}$ & $3.5 \mathrm{a}$ & $3.5 \mathrm{~b}$ \\
\hline 5 & $3.7 \mathrm{a}$ & $3.7 \mathrm{a}$ & $3.8 \mathrm{a}$ & $3.5 \mathrm{~b}$ \\
\hline 7 & $3.5 \mathrm{a}$ & $3.3 \mathrm{a}$ & $4.2 \mathrm{a}$ & $4.5 \mathrm{a}$ \\
\hline \multicolumn{5}{|l|}{42 WAP } \\
\hline 3 & $3.0 \mathrm{a}$ & $3.2 \mathrm{a}$ & $3.8 \mathrm{~b}$ & $3.3 \mathrm{~b}$ \\
\hline 5 & $3.5 \mathrm{a}$ & $3.5 \mathrm{a}$ & $3.8 \mathrm{~b}$ & $3.5 \mathrm{~b}$ \\
\hline 7 & $3.3 \mathrm{a}$ & $3.3 \mathrm{a}$ & $4.6 \mathrm{a}$ & $4.2 \mathrm{a}$ \\
\hline \multicolumn{5}{|l|}{48 WAP } \\
\hline 3 & $2.5 \mathrm{a}$ & $3.2 \mathrm{a}$ & $3.7 \mathrm{a}$ & $3.3 \mathrm{ab}$ \\
\hline 5 & $3.3 \mathrm{a}$ & $3.7 \mathrm{a}$ & $4.0 \mathrm{a}$ & $3.2 \mathrm{~b}$ \\
\hline 7 & $3.6 \mathrm{a}$ & $3.8 \mathrm{a}$ & $4.3 \mathrm{a}$ & $4.5 \mathrm{a}$ \\
\hline \multicolumn{5}{|l|}{54 WAP } \\
\hline 3 & $2.7 \mathrm{a}$ & $3.2 \mathrm{~b}$ & $3.3 \mathrm{a}$ & $3.5 \mathrm{a}$ \\
\hline 5 & $3.0 \mathrm{a}$ & $3.7 \mathrm{ab}$ & $3.5 \mathrm{a}$ & $3.7 \mathrm{a}$ \\
\hline 7 & $3.3 \mathrm{a}$ & $4.5 \mathrm{a}$ & $4.0 \mathrm{a}$ & $4.3 \mathrm{a}$ \\
\hline
\end{tabular}

${ }^{2} \mathrm{WAP}=$ week after planting; $1 \mathrm{lb} / 1000 \mathrm{ft}^{2}=48.8243 \mathrm{~kg} \cdot \mathrm{ha}^{-1}$

${ }^{y} 0=$ dead plant; 5 = outstanding plant quality (dense leaf canopy, high-quality flowers, and no nutrient deficiencies or dieback).

'Mean separation for each species and WAP by Tukey's honestly significant difference test at $P<0.05$.

and normality plots of the conditional residuals.

\section{Results and discussion}

Evaluation of $\mathbf{N}$ FerTilizer REQUIREMENTS. In previous research conducted with annuals, perennials, and woody shrubs at the same test site in west-central Florida, we observed that plant growth increased in response to increasing $\mathrm{N}$ fertilizer rate (Shober et al., 2013; Shurberg et al., $2012 a, b)$. We also observed that acceptable quality plants were maintained with lower $\mathrm{N}$ fertilizer rates. Since the goal of this study was to identify $\mathrm{N}$ fertilizer rates (based on previous studies) that would produce and maintain acceptable quality vines and groundcovers, growth response to $\mathrm{N}$ fertilizer rate was not included as part of this study.

Fertilizer rate had no effect on quality of pipe vine or coral honeysuckle at any time throughout the study (Table 1). Both species were consistently rated as below average quality after $12 \mathrm{WAP}$, regardless of $\mathrm{N}$ fertilizer rate. All coral honeysuckle were dead by 36 WAP because of disease (Table 1). Pipe vine suffered from cold damage early in the study (6 WAP) and several plants were dead by $12 \mathrm{WAP}$. Surviving pipe vine plants maintained quality ratings above 3.0 through 54 WAP when fertilized at an annual $\mathrm{N}$ rate of $2 \mathrm{lb} / 1000 \mathrm{ft}^{2}$ or higher (data not shown). However, the high quality of surviving plants was not evident in overall quality ratings because of the influence of dead plants, which were assigned a value of 0 for analysis (Table 1 ). In contrast, cross vine $(36,42$, and 54 WAP), african iris (48 to 54 WAP), and society garlic (18 to 24 WAP) plants fertilized at higher annual $\mathrm{N}$ rates $\left(12 \mathrm{lb} / 1000 \mathrm{ft}^{2}\right)$ had higher quality ratings than plants fertilized at 0 or $2 \mathrm{lb} / 1000 \mathrm{ft}^{2}$ of $\mathrm{N}$ per year (Tables 1 and 2). For species that responded to $\mathrm{N}$ fertilization, acceptable quality ratings of 3 or better were achieved when plants were supplied with at least $4 \mathrm{lb} / 1000 \mathrm{ft}^{2}$ per year $\mathrm{N}$ (Tables 1 and 2). On the basis of these results, we suggested annual $\mathrm{N}$ fertilization with 2 to $4 \mathrm{lb} / 1000 \mathrm{ft}^{2}$ would be adequate to maintain good quality vines and groundcovers when grown in west-central Florida.

VALIDATION OF $\mathbf{N}$ FerTILIZER RECOMMENDATIONS. Additional species were planted in the landscape to determine if the recommended 2 to $4 \mathrm{lb} / 1000 \mathrm{ft}^{2}$ annual $\mathrm{N}$ rate was adequate for additional groundcovers and vines. Only carolina yellow jessamine plants (42 WAP) and passion vine plants (30 through 48 WAP) were rated as having higher quality when they received $7 \mathrm{lb} / 1000 \mathrm{ft}^{2}$ of $\mathrm{N}$ annually compared with plants that were supplied with $\mathrm{N}$ at 3 or 5 $\mathrm{lb} / 1000 \mathrm{ft}^{2}$ annual rate; both species maintained acceptable quality ratings of 3 or better at all $\mathrm{N}$ fertilizer rates (Table 3). Ornamental sweetpotato and purple lovegrass were consistently rated as below average quality $(<3)$ after 18 WAP (Table 4) at all $\mathrm{N}$ fertilizer rates, suggesting factors other than $\mathrm{N}$ fertilization were affecting plant quality. In the case of ornamental sweetpotato, most vines were impacted by the presence of tortoise beetle (Charidotella bicolor), which caused defoliation and negatively affected plant quality. Purple lovegrass were cut back at 24 WAP to remove dead tissue after cold damage; many plants did not fully recover.

Discussion. Few studies exist in the literature that evaluated the $\mathrm{N}$ fertilizer rates that produce acceptable quality vines and groundcovers in the landscape. Stafne et al. (2005) evaluated the effects of $\mathrm{N}$ fertilizer rate on establishment of creeping 
Table 4. Average plant quality response of additional landscape-grown groundcover species to controlled release nitrogen $(\mathrm{N})$ applied at three rates planted 15 Aug. 2011 in west-central Florida (U.S. Department of Agriculture hardiness zone $9 \mathrm{~b}$ ) to validate $\mathrm{N}$ fertilizer recommendations.

\begin{tabular}{|c|c|c|c|c|}
\hline \multirow[b]{2}{*}{$\begin{array}{l}\text { WAP and fertilizer } \\
\mathrm{N} \text { rate }\left(\mathrm{lb} / 1000 \mathrm{ft}^{2}\right)^{\mathrm{z}}\end{array}$} & \multicolumn{4}{|c|}{ Plant aesthetic quality $(0-5 \text { scale })^{\mathrm{y}}$} \\
\hline & Juniper & $\begin{array}{c}\text { Sunshine } \\
\text { mimosa }\end{array}$ & $\begin{array}{c}\text { Purple } \\
\text { lovegrass }\end{array}$ & $\begin{array}{c}\text { Ornamental } \\
\text { sweetpotato vine }\end{array}$ \\
\hline \multicolumn{5}{|l|}{$6 \mathrm{WAP}$} \\
\hline 3 & $4.1 \mathrm{a}^{\mathrm{x}}$ & $4.1 \mathrm{a}$ & $3.9 \mathrm{a}$ & $4.4 \mathrm{a}$ \\
\hline 5 & $4.6 \mathrm{a}$ & $4.2 \mathrm{a}$ & $4.2 \mathrm{a}$ & $4.2 \mathrm{a}$ \\
\hline 7 & $4.1 \mathrm{a}$ & $4.4 \mathrm{a}$ & $4.2 \mathrm{a}$ & $4.7 \mathrm{a}$ \\
\hline \multicolumn{5}{|l|}{$12 \mathrm{WAP}$} \\
\hline 3 & $4.3 \mathrm{a}$ & $4.7 \mathrm{a}$ & $4.0 \mathrm{a}$ & $3.3 \mathrm{a}$ \\
\hline 5 & $4.4 \mathrm{a}$ & $4.7 \mathrm{a}$ & $4.4 \mathrm{a}$ & $4.0 \mathrm{a}$ \\
\hline 7 & $4.4 \mathrm{a}$ & $4.4 \mathrm{a}$ & $4.2 \mathrm{a}$ & $3.7 \mathrm{a}$ \\
\hline \multicolumn{5}{|l|}{18 WAP } \\
\hline 3 & $4.3 \mathrm{a}$ & $4.6 \mathrm{a}$ & $2.7 \mathrm{a}$ & $2.8 \mathrm{a}$ \\
\hline 5 & $4.7 \mathrm{a}$ & $4.8 \mathrm{a}$ & $3.0 \mathrm{a}$ & $2.9 \mathrm{a}$ \\
\hline 7 & $4.1 \mathrm{a}$ & $4.9 \mathrm{a}$ & $2.9 \mathrm{a}$ & $3.1 \mathrm{a}$ \\
\hline \multicolumn{5}{|l|}{24 WAP } \\
\hline 3 & $3.8 \mathrm{a}$ & $3.8 \mathrm{a}$ & $2.4 \mathrm{a}$ & $2.0 \mathrm{a}$ \\
\hline 5 & $4.2 \mathrm{a}$ & $3.9 \mathrm{a}$ & $2.9 \mathrm{a}$ & $1.4 \mathrm{a}$ \\
\hline 7 & $4.0 \mathrm{a}$ & $4.1 \mathrm{a}$ & $2.2 \mathrm{a}$ & $2.0 \mathrm{a}$ \\
\hline \multicolumn{5}{|l|}{$30 \mathrm{WAP}$} \\
\hline 3 & $3.8 \mathrm{a}$ & $4.2 \mathrm{a}$ & $2.1 \mathrm{a}$ & $2.1 \mathrm{a}$ \\
\hline 5 & $4.1 \mathrm{a}$ & $4.4 \mathrm{a}$ & $2.3 \mathrm{a}$ & $1.9 \mathrm{a}$ \\
\hline 7 & $4.0 \mathrm{a}$ & $4.6 \mathrm{a}$ & $2.4 \mathrm{a}$ & $2.6 \mathrm{a}$ \\
\hline \multicolumn{5}{|l|}{36 WAP } \\
\hline 3 & $3.7 \mathrm{a}$ & $4.9 \mathrm{a}$ & $2.4 \mathrm{a}$ & $2.9 \mathrm{a}$ \\
\hline 5 & $4.3 \mathrm{a}$ & $4.9 \mathrm{a}$ & $2.8 \mathrm{a}$ & $3.1 \mathrm{a}$ \\
\hline 7 & $4.0 \mathrm{a}$ & $5.0 \mathrm{a}$ & $2.8 \mathrm{a}$ & $3.3 \mathrm{a}$ \\
\hline \multicolumn{5}{|l|}{$42 \mathrm{WAP}$} \\
\hline 3 & $3.8 \mathrm{a}$ & $5.0 \mathrm{a}$ & $2.4 \mathrm{a}$ & $2.3 \mathrm{a}$ \\
\hline 5 & $3.9 \mathrm{a}$ & $4.0 \mathrm{a}$ & $2.8 \mathrm{a}$ & $2.4 \mathrm{a}$ \\
\hline 7 & $4.6 \mathrm{a}$ & $5.0 \mathrm{a}$ & $2.8 \mathrm{a}$ & $2.8 \mathrm{a}$ \\
\hline \multicolumn{5}{|l|}{$48 \mathrm{WAP}$} \\
\hline 3 & $3.7 \mathrm{a}$ & $5.0 \mathrm{a}$ & $2.6 \mathrm{a}$ & $1.9 \mathrm{a}$ \\
\hline 5 & $4.1 \mathrm{a}$ & $5.0 \mathrm{a}$ & $2.7 \mathrm{a}$ & $2.6 \mathrm{a}$ \\
\hline 7 & $3.9 \mathrm{a}$ & $5.0 \mathrm{a}$ & $2.8 \mathrm{a}$ & $2.8 \mathrm{a}$ \\
\hline \multicolumn{5}{|l|}{$54 \mathrm{WAP}$} \\
\hline 3 & $3.7 \mathrm{a}$ & $5.0 \mathrm{a}$ & $2.8 \mathrm{a}$ & $1.7 \mathrm{a}$ \\
\hline 5 & $3.8 \mathrm{a}$ & $4.9 \mathrm{a}$ & $3.2 \mathrm{a}$ & $2.7 \mathrm{a}$ \\
\hline 7 & $3.8 \mathrm{a}$ & $5.0 \mathrm{a}$ & $3.0 \mathrm{a}$ & $2.7 \mathrm{a}$ \\
\hline
\end{tabular}

${ }^{2} \mathrm{WAP}=$ week after planting; $1 \mathrm{lb} / 1000 \mathrm{ft}^{2}=48.8243 \mathrm{~kg} \cdot \mathrm{ha}^{-1}$.

${ }^{\mathrm{y}} 0=$ dead plant; 5 = outstanding plant quality (dense leaf canopy, high-quality flowers, and no nutrient deficiencies or dieback).

${ }^{x}$ Mean separation for each species and WAP by Tukey's honestly significant difference test at $P<0.05$

lilyturf (Liriope spicata), english ivy, and 'Coloratus' purpleleaf wintercreeper euonymus (Euonymus fortunei) grown in sun and shade in Arkansas. Plants received 1, 2, 3, or 4 applications of $6 \mathrm{oz}$ of $13 \mathrm{~N}-5.67 \mathrm{P}-$ $10.8 \mathrm{~K}$ fertilizer per $25 \mathrm{ft}^{2}$ bed (equivalent to an annual $\mathrm{N}$ application rate of $2.14,4.28,6.42$, or $8.56 \mathrm{lb} / 1000 \mathrm{ft}^{2}$ ). The authors reported no effect of fertilizer treatment on growth or establishment of any species at 6 months after the first fertilizer application (three fertilizer applications had been applied by 6 months after planting). Euonymus had greater fresh and dry shoot weights at 1 year after planting when they received four fertilizer applications, compared with 1, 2, or 3 fertilizer applications. However, the authors noted that all three species produced acceptable growth under all fertilizer treatments. No fertilizer rate effects on percent canopy cover were documented for any of the three species under any fertilizer treatment (Stafne et al., 2005). In a series of greenhouse experiments, Holcomb et al. (1992) reported satisfactory growth and no difference in growth for 'Baltica' baltic ivy $(H$. helix) fertilized with 50 and 100 $\mathrm{mg} \cdot \mathrm{L}^{-1} \mathrm{~N}$ per month for 12 weeks as either $20 \mathrm{~N}-4.4 \mathrm{P}-16.6 \mathrm{~K}$ or $20 \mathrm{~N}-$ $8.4 \mathrm{P}-14.9 \mathrm{~K}$ fertilizer during production in $11-\mathrm{cm}$ pots (equivalent to $\mathrm{N}$ application at a rate of 0.79 and 1.61 $\mathrm{lb} / 1000 \mathrm{ft}^{2}$ per application). Our data support some of the previous work that acceptable quality vines and groundcovers can be maintained in the landscape with lower $\mathrm{N}$ fertilizer rates.

\section{Conclusions}

We acknowledge that response to $\mathrm{N}$ fertilization will likely vary under different soil and environmental conditions. However, in central Florida annual $\mathrm{N}$ applications of 3 to $4 \mathrm{lb} /$ $1000 \mathrm{ft}^{2} \mathrm{~N}$ produced acceptable quality plants with ratings of 3 or better in most cases although increasing $\mathrm{N}$ fertilization rate did produce higher quality vine and groundcover plants for some species. On the basis of these results and supported by the limited published research on response of vine and groundcover species to $\mathrm{N}$ fertilization, we suggest that fertilization with 2 to $4 \mathrm{lb} / 1000 \mathrm{ft}^{2}$ per year should be adequate to maintain acceptable vines and groundcovers grown in west-central Florida, noting that results may vary by species and location. Future research should focus on the quality response of vines and groundcovers to $\mathrm{N}$ fertilizer under varying soil and environmental conditions, as well as the environmental and economic benefits of reducing $\mathrm{N}$ fertilization.

\section{Literature cited}

Berberich, S., J. Snyder, R. Geneve, and M.A. Williams. 2006. Growth and flowering response of container grown passion flower cultivars to fertilizer and paclobutrazo. J. Environ. Hort. 24: 109-114.

Dumroese, R.K. 2003. Growth of Juniperus and Potentilla using liquid exponential and controlled-release fertilizers. HortScience 38:1378-1380.

Florida Friendly Landscaping Program. 2009. The Florida Yards \& Neighborhoods Handbook. Univ. Florida Inst. Food Agr. Sci., Gainesville, FL.

Henley, R.W. and R.J. Black. 2003. English ivies to know and grow. Univ. Florida Inst. Food Agr. Sci., Gainesville, FL. 
Holcomb, E.J., S. Gamez, D. Beattie, and G.C. Elliott. 1992. Efficiency of fertigation programs for baltic ivy and asiatic lily. HortTechnology 2:43-46.

Park-Brown, S. and G.W. Knox. 2010. Flowering vines for Florida. Univ. Florida Inst. Food Agr. Sci., Gainesville, FL.

Santos, B.M. and J.P. Gilreath. 2006. Influence of nitrogen fertilization and support systems on passion fruit yield and economic feasibility. HortTechnology 16:43-45.

Shober, A.L., K.A. Moore, N.G. West, C. Wiese, G. Hasing, G. Denny, and G.W. Knox. 2013. Growth and quality response of woody shrubs to nitrogen fertilization rates during landscape establishment in Florida. HortTechnology 23:898-904.
Shober, A.L., S. Davis, M.D. Dukes, G.C. Denny, S.P. Brown, and S. Vyapari. 2009. Performance of Florida landscape plants when irrigated by ET-based controllers and time-based methods. J. Environ. Hort. 27:251-256.

Shurberg, G., A.L. Shober, C. Wiese, G. Denny, G.W. Knox, K.A. Moore, and M.C. Giurcanu. 2012a. Growth and quality response of five landscape-grown herbaceous perennials to nitrogen fertilization at five rates. HortTechnology 22:787-797.

Shurberg, G., A.L. Shober, C. Wiese, G. Denny, G.W. Knox, K.A. Moore, and M.C. Giurcanu. 2012b. Response of landscape-grown warm- and cool-season annuals to nitrogen fertilization at five rates. Hort Technology 22:368-375.
Stafne, R., A. Einert, and G. Klingaman. 2005. Fertilizer applications on establishment and growth of three groundcover species in sun and shade. J. Environ. Hort. 23:157-161.

U.S. Department of Agriculture. 2004. Official soil series descriptions. U.S. Dept. Agr. Natural Resources Conservation Serv., Washington, DC.

Utsunomiya, N., H. Kinai, Y. Matsui, and T. Takebayashi. 1998. The effects of chitosan oligosaccharides soil conditioner and nitrogen fertilizer on the flowering and fruit growth of purple passionfruit (Passiflora edulis Sims var. edulis). J. Jpn. Soc. Hort. Sci. 67:567-571. 